\title{
Las promesas en el ámbito de la salud pública, elecciones presidenciales 2018, Costa Rica
}

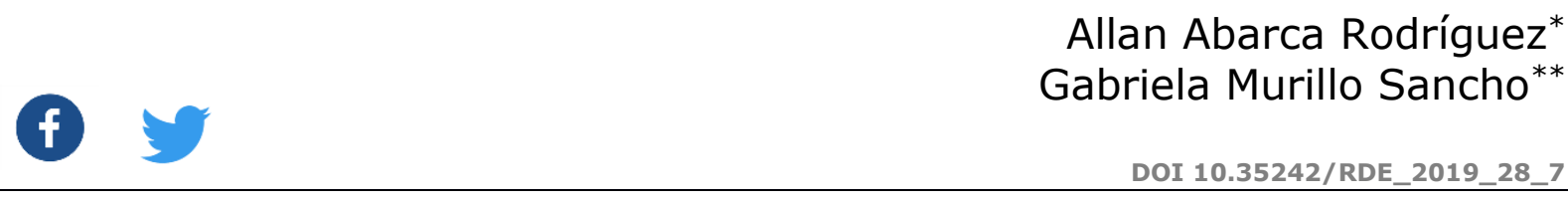

Nota del Consejo Editorial

Recepción: 7 noviembre de 2019.

Revisión, corrección y aprobación: 14 de junio de 2019.

Resumen: El análisis de las propuestas de política pública ofertadas en una campaña electoral presidencial resulta esencial para determinar los problemas públicos que se abordarán en una posible gestión de gobierno. Este artículo analiza las promesas realizadas en la campaña presidencial 2018, en Costa Rica, en el ámbito de la salud. Se concluye, respecto de una diversidad de ejes temáticos presentados, que enfatiza en propuestas sobre: a) la gestión en salud y la prestación de los servicios de salud y b) las políticas de promoción de la salud, prevención y educación para la salud. No obstante, sobresalen también propuestas sobre el recurso hídrico, la vialidad (concentrada en políticas para aminorar los accidentes de tránsito y mejorar la transitabilidad por las ciudades), y políticas sobre sexualidad (lo que incluye salud sexual y derechos sexuales y/o reproductivos).

Palabras clave: Promesas de campaña / Compromisos políticos / Programas de gobierno / Campaña política / Salud pública / Política de salud / Política pública.

Abstract: The analysis of public policy proposals presented during a presidential election campaign is essential to determine the public issues that will be addressed in a possible president's term. This article analyzes the promises, regarding health topics, made during the 2018 Costa Rican presidential campaign. Given a diversity of thematic axes, this article concludes that there is an emphasis on: a. health management and the provision of health services; and b. health promotion and prevention and health education policies. However, proposals about water resources, road infrastructure (focusing on traffic accident reduction and improving transit within cities), and policies concerning sexuality (including sexual health, and sexual and/or reproductive rights) standout as well.

Key Words: Campaign promise / Political commitments / Government programs / Political campaign / Public health / Health policy / Public policy.

\footnotetext{
* Costarricense, politólogo y especialista en políticas públicas, correo allan.abarca@ucr.ac.cr. Profesor catedrático de la Universidad de Costa Rica. Se desempeña como docente-investigador en la Escuela de Salud Pública. Tiene una maestría en Gobierno y Asuntos Públicos, una maestría en Salud Pública, y es egresado de la Maestría en Estudios Interdisciplinarios de Discapacidad. Las investigaciones que realiza se enfocan en análisis de la formulación y ejecución de políticas públicas.

** Costarricense, educadora y psicopedagoga, correo maria.murillo@ucr.ac.cr. Profesora asociada de la Universidad de Costa Rica. Es docente-investigadora de la Escuela de Salud Pública. Cuenta con una maestría en Psicopedagogía y es egresada de la Maestría en Salud Pública con énfasis en Gerencia de la Salud. Los temas de investigación incluyen educación superior, desempeño docente, promoción de la salud, ciudades saludables, objetivos del desarrollo sostenible.
} 


\section{DERECHO ELECTORAL}

\section{INTRODUCCIÓN}

En febrero del año 2018 se realizaron las elecciones nacionales en Costa Rica. Acude a las urnas el $65,7 \%$ del electorado inscrito quien elige al presidente(a) de la República y los miembros que compondrán la Asamblea Legislativa (diputados). Para la presidencia de la República se inscribieron 13 candidaturas (12 hombres y 1 mujer). Dado que ningún partido político logró el mínimo necesario (40\%) y los que obtuvieron mayor porcentaje fueron el partido Restauración Nacional (PRN) con un $24,99 \%$ y el partido Acción Ciudadana (PAC) con un $21,63 \%$, se efectuó una segunda ronda en abril de 2018, con los candidatos de ambos partidos; el resultado fue de un 60,59\% para el Partido Acción Ciudadana (TSE, 2018).

Desde el inicio de la campaña electoral, el Tribunal Supremo de Elecciones (TSE) lanza una campaña publicitaria para invitar al voto informado, esto es, un llamado a conocer las propuestas de los candidatos; se denominó "Un Voto Informado es un Voto Inteligente", con un costo de $\not 464.010 .000,00$ (Castro, comunicación personal vía correo electrónico, 27 de agosto de 2018), lo que aproximadamente son $\$ 111.498,20$ al tipo de cambio vigente el 4 de febrero de 2018.

Esta campaña fue presentada en setiembre del año 2017 con la intención de auspiciar un voto informado en las personas; esto es, que el ejercicio de una ciudadanía responsable implica informarse antes de votar, para el beneficio colectivo (La Revista, 2017). La campaña tuvo un enfoque motivacional (el llamado a votar con conciencia) y otro informativo (logística para ejercer el voto). El presidente del TSE, Dr. Luis A. Sobrado indica que "con el programa votante informado hemos tratado de dar mucha exposición a los candidatos y hemos apoyado todo tipo de encuentros y debates" (Semanario Universidad, 2018).

Si bien es en enero de 2018 cuando se intensifica la difusión de los programas de gobierno -dada la mayor inversión publicitaria de los partidos políticos y la ejecución de múltiples debates-, la coyuntura electoral fue marcada por la resolución de la Corte Interamericana de Derechos Humanos (CIDH) ante la opinión consultiva realizada por el Gobierno de la República de Costa Rica, respecto al cambio de nombre y sexo de las personas a partir de su identidad de género y "¿si es necesaria la existencia de una figura jurídica que regule los vínculos entre personas del mismo sexo, para que el Estado reconozca todos los derechos patrimoniales que se derivan de esta relación?" (CIDH, 2017), entre otros aspectos consultados. 


\section{DERECHO EIECTORAL}

El criterio de la administración Solís Rivera (2014-2018) de reconocer a las uniones legales de personas del mismo sexo los derechos legislados para parejas heterosexuales, incluido el derecho al matrimonio, generó una campaña cuasi monotemática.

En efecto, el debate en torno a las políticas públicas necesarias para el país, presentes en las distintas propuestas programáticas, queda relegado para dar paso al centralismo del tema religioso, lo que Rosales (2018, p. 61) denomina "shock religioso". Por eso, Díaz postula el criterio de que "si el crecimiento electoral de Alvarado Muñoz (PRN) fue una reacción a la resolución de la $\mathrm{CIDH}$, el de Alvarado Quesada (PAC) parece haber sido una respuesta al crecimiento del PRN" (2018, p. 39).

Rosales (2018) precisa que la campaña política se caracterizó por una diversidad de partidos políticos (con candidaturas muy diferentes entre sí en cuanto a edad, trayectoria política, posición ideológica y religiosa) y lo que denomina la volatilidad de la participación ciudadana en el proceso. Sobre este último aspecto -la volatilidad-, Díaz (2018, p. 38) indica que responde a un desencanto y deslealtad partidaria, así como a la fragmentación interna en los partidos políticos del momento.

Para la segunda ronda electoral, Rosales (2018, p. 64) destaca, entre otros, el surgimiento de "Coalición por Costa Rica", creada con el fin de un frente común por los derechos humanos, entre otros temas. Para Díaz (2018), la apuesta por un "gobierno de unidad nacional" pareciera que resultó una mejor opción para hacer frente a situaciones de gobernabilidad.

De la campaña política se esperaba un debate sobre temas centrales de la salud que habían estado en la agenda pública los últimos dos años: conflictos sobre el acceso al agua, deficiencia en la atención en los servicios de salud (particularmente reflejado en las listas de espera), agotamiento del sistema de pensiones, políticas sobre gestión de residuos, el asfixiante colapso vial, entre otros.

Bajo el reconocimiento de que el componente salud es fundamental en el desarrollo y la calidad de vida de una nación, este artículo tiene como propósito un análisis de las propuestas de los partidos políticos, expresadas en la campaña electoral 2018, clasificables en el ámbito de la salud pública. 


\section{DERECHO ELECTORAL}

\section{Metodología}

La unidad de observación es el programa de gobierno de los partidos políticos (participantes de la primera ronda). A partir de esta, se inicia un análisis que comprende identificar y clasificar aquellas promesas políticas (oferta de política pública) que son propias del ámbito de la salud pública.

Solamente 3 programas de gobierno incluyen una sección o acápite específico sobre salud, en estos casos se aborda cada una de las promesas; también se analizan las otras secciones para detectar aquellas promesas que son del ámbito de la salud pública. Para los otros 10 programas de gobierno, se analiza cada propuesta de política pública a fin de identificar las propias de la salud o bien que están intrínsecamente ligadas a problemas de salud. Por ejemplo, hay temas que son propios de la salud, aunque estén ubicados en otros ámbitos: políticas públicas respecto del bullying y de la violencia doméstica podrían estar localizadas en secciones relativas a "política educativa" y a "política social"; dado que también son propias de la salud pública, se les retoma como tales.

Cuando se detecta una promesa en el ámbito de la salud se procede a clasificarla en un descriptor o categoría, por ejemplo, "bullying", "promoción de la salud", "prevención de la salud", "gestión de residuos y reciclaje", entre otras. No obstante, es posible que una propuesta de política pública, según el propósito de su enunciado, se sitúe en dos ámbitos temáticos a la vez, por ejemplo, en "derecho a la salud" y en "primer nivel de atención". Cuando sucede esto último se procede a clasificarla en ambas para tener claros los ámbitos de intervención de las políticas, aunque se reconoce que solo es una propuesta. En concreto, interesa tanto la frecuencia de las políticas públicas anunciadas en salud como los ámbitos propios de intervención de cada política.

Hay ofertas políticas como la "infraestructura" o la "generación de empleo", que si bien tienen un impacto en la salud de las personas -son determinantes sociales de la salud-; por el hecho de que se presentan como soluciones exclusivas de la reactivación económica del país, se prefiere no incluirlas como políticas de salud. Algunos temas, si bien están relacionados con la salud pública, se omite darles un seguimiento y análisis detallado, por ejemplo, "energías limpias" y "áreas silvestres", ya que tienen un estrecho vínculo con el macrotema "asuntos ambientales". En cambio, siendo el "reciclaje y gestión de residuos" temas ambientales, pero 


\section{DERECHO ELECTORAL}

altamente sensibles y de discusión permanente en el ámbito de la salud pública, sí se les considera.

Así, la idea de construir una carretera por sí sola no se clasifica en "salud", a no ser que la solución esté claramente auspiciada en el marco de un problema de salud pública, por ejemplo, los referidos a "movilidad urbana". Este último tema ha sido central en la discusión pública de problemas adversos a la salud en Costa Rica.

Para la determinación del millar de promesas cuantificadas (véase el segmento "3.1 De las promesas realizadas"), se procedió a estructurar una base de datos en la cual la variable de interés principal era la promesa. Se añadieron dos variables: la existencia o no de metas para la política y su posible expresión problemática. El diseño de base de datos pretendió evitar la confusión de una promesa con una meta, esto para no generar el sesgo de la amplificación en el número de las ofertas electorales.

Así pues, a una promesa concreta (por ejemplo, "tercerizar los servicios de salud que se puedan obtener más baratos en el sector privado") se le clasificó en un ámbito en particular, esto es, el descriptor más próximo (en el caso ejemplificado corresponde a "servicios de salud"). Esta primera clasificación se ejecutó a partir de los temas y áreas propias de la salud expuestas en los informes mundiales de la salud de los años 2007, 2008 y 2010, además de los ámbitos de salud definidos en la Conferencia Internacional sobre Promoción de la Salud de Ottawa (1986), de la Conferencia Internacional sobre la Promoción de la Salud de Yakarta (1997) y de los problemas públicos principales en materia de salud congruentes con la definición de la salud de la Organización Mundial de la Salud (OMS).

Precisamente, el enunciado de salud, escrito en el preámbulo de la OMS del año 1946, establece una concepción integral, suponiendo que "es un estado de completo bienestar físico, mental y social, y no solamente la ausencia de afecciones o enfermedades". A partir de esta circunstancia, aspectos como la contaminación, la bioética, la violencia infantil, las ciclovías, las pensiones, la atención médica, entre otros, forman parte de los problemas y retos propios de la salud.

Por lo anterior, con la clasificación inicial de identificación de promesas, el objetivo es dar cuenta del amplio abanico temático con el cual se auspician promesas de salud y, de estas, cuáles son en concreto las que promueven los partidos políticos, como parte de una eventual agenda de gobierno. 


\section{DERECHO ELECTORAL}

Las promesas de campaña en salud abarcan la siguiente tipología: acreditación de los servicios de salud, rectoría del Ministerio de Salud, promoción de la salud, salud preventiva, participación comunal en salud, agua, investigación científica en salud, planificación de servicios, organización de servicios de salud, comunicación de políticas en salud, atención en salud, sistema y gestión en salud, calidad de los servicios, inter-institucionalidad para políticas de salud, sistemas de información en salud, gestión de residuos y reciclaje, vigilancia de la salud, reestructuración de servicios de salud, reorganización de servicios de salud, recursos humanos para salud, medicamentos, sistema de salud, TIC en salud, formación continua en salud, educación para la salud, finanzas de la Caja Costarricense de Seguro Social (CCSS), primer nivel de atención, aseguramiento, equidad y acceso, atención primaria, riesgos y vulnerabilidad, listas de espera, derechos a la salud, salud comunitaria, salud sexual y salud sexual reproductiva, adulto mayor y salud, niñez y salud, mujer y salud, indígenas y salud, centros de cuido en salud, deporte, recreación, ciudades saludables, servicios de salud, movilidad urbana, discapacidad y salud, pensiones, vialidad, ciclovías, seguridad vial y accidentes, adicciones, bullying, salud mental, salud global, violencia de género, violencia intradomiciliaria, violencia infantil, adicciones, cáncer, rehabilitación, vulnerabilidad y salud, infraestructura, riesgo de trabajo, violencia contra el adulto mayor, salud ocupacional, ciudades amigables, seguridad y salud, contaminación, transparencia en acciones e instituciones de la salud, bioseguridad, bioética, modelos de salud, planificación urbana, derecho laboral y otros temas en salud no incluidos en los anteriores.

Finalmente, una vez identificadas las promesas políticas por partido en el ámbito de la salud, se procede a definir categorías amplias en las que se agrupan, de manera que se obtiene una primera clasificación de grandes temáticas. Con la reagrupación se pretende generar núcleos de representación homogénea que recojan de mejor manera el énfasis general del conjunto de promesas en salud de cada partido político. Por ejemplo, hay promesas temáticas en "dirección y planificación de servicios de salud", "gestión de la atención en salud", "reestructuración de los servicios de salud", "reorganización de los servicios de salud", "recursos humanos para la atención en salud"; no obstante, se pueden reagrupar en el núcleo mayor denominado "sistema y servicios de salud". Con esto hay una visión general más compacta acerca de los grupos de problemas públicos que en materia de salud se privilegian en el país, al menos en el discurso y propuesta de los partidos políticos partícipes en la contienda electoral para la presidencia de la República. 


\section{DERECHO EIECTORAL}

En otras palabras, con la reagrupación temática es posible generar un perfil general más entendible de los énfasis programáticos o de intervención que en materia de salud proponen los partidos políticos.

\section{Resultados}

\subsection{De LAS PROMESAS REALIzAdas}

En el ámbito de la salud, los 13 partidos políticos esbozan un total de 1052 propuestas políticas. El Partido Acción Ciudadana (PAC) y el Partido Unidad Social Cristiana (PUSC) se constituyen en los mayores oferentes con 161 promesas cada uno. Este último partido, que es cuarto en el proceso electoral, tuvo de candidato a un expresidente ejecutivo de la Caja Costarricense de Seguro Social (CCSS) durante el período 1998-2002, institución que brinda los servicios de salud públicos.

Destaca el hecho de que el partido que terminó de primero en la primera ronda electoral, el Partido Restauración Nacional, es apenas el octavo en promesas en materia de salud, esto es, con una reducida oferta de políticas como se puede observar en la figura 1.

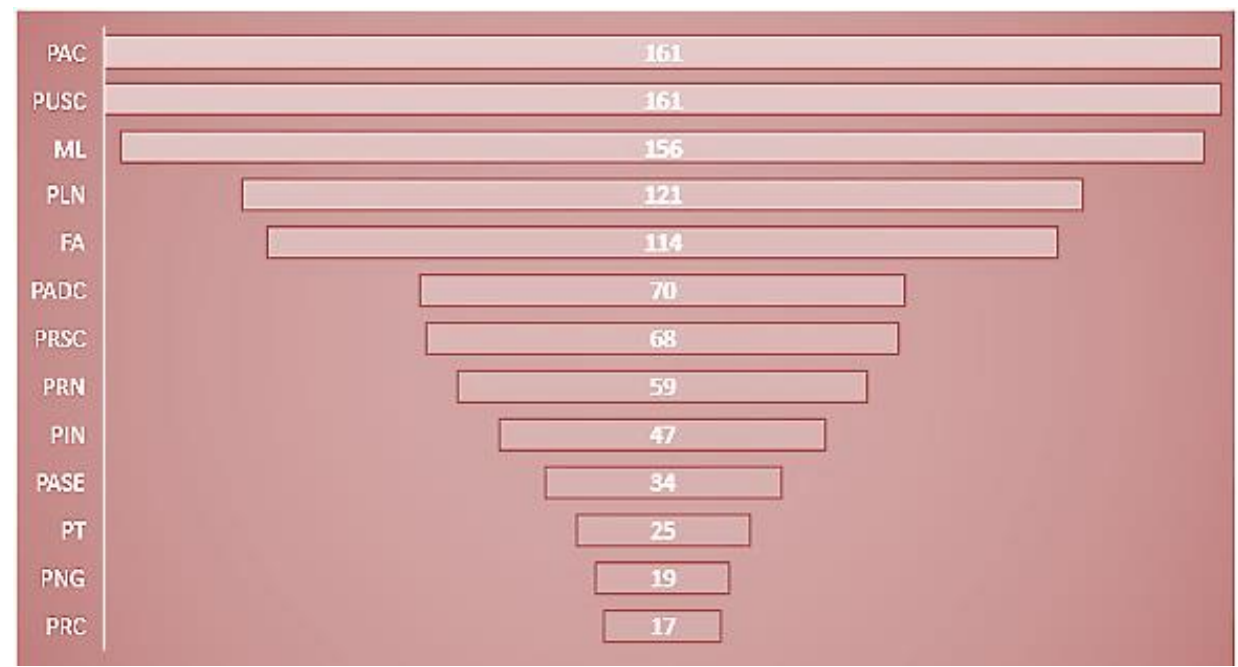

Figura 1. Promesas de políticas en el campo de la salud, elecciones presidenciales 2018 , según partido político, Costa Rica. Elaborado con base en los programas de gobierno.

Notas: PAC Partido Acción Ciudadana, PUSC Partido Unidad Social Cristiana, ML Movimiento Libertario, PLN Partido Liberación Nacional, FA Frente Amplio, PADC Partido Alianza Demócrata Cristiana, PRSC Partido Republicano Social Cristiano, PRN Partido Renovación Nacional, PIN Partido Integración Nacional, PASE Partido Accesibilidad Sin Exclusión, PT Partido de los Trabajadores, PNG Partido Nueva Generación, PRC Partido Renovación Costarricense. 


\section{DERECHO ELECTORAL}

Las propuestas son variadas y se encuentran distribuidas en 75 temas identificados, los cuales incluyen un amplio abanico de políticas, por ejemplo, enfermedades (cáncer), rectoría del Ministerio de Salud, derecho a la salud, atención al adulto mayor, políticas de recreación, políticas de salud mental, red vial, adicciones, entre otros.

\section{2 ÉNFASIS TEMÁTICO DE LAS PROMESAS}

En términos agregados (considerando las políticas independientemente del partido político que las propuso) las "políticas públicas del deporte" se sitúan en el primer lugar, lo cual llama la atención por cuanto el sector ha sido tradicionalmente relegado de las políticas del Estado. Recuérdese, por ejemplo, cuando el ministro de Deportes, William Todd, anunció en el año 2011 la posibilidad de una teletón para financiar el deporte, aduciendo la disponibilidad de un "escasísimo recurso económico" por parte del Gobierno (La Nación).

En segundo lugar, destacan las "políticas sobre el recurso hídrico", lo cual demuestra la preocupación que el tema ha posicionado en la agenda pública, y que el propio Estado de la Nación (2017) ha mencionado, destacando aspectos tales como el poco tratamiento de aguas residuales y la desmejora en la calidad de los cuerpos de agua, además de la referencia a los procesos agrícolas que amenazan la calidad del agua, la falta de servicio de agua o el acceso al agua potable, la insostenibilidad en el uso de recursos naturales clave para el desarrollo y protección del agua, así como la inadecuada o inexistente gestión de residuos sólidos y líquidos en relación con los cuerpos de agua.

En tercer lugar, políticas vinculadas a la reestructuración y reorganización de servicios, programas y de instituciones del campo de la salud. En cuarto lugar, políticas en promoción de la salud. Según la figura 2 respecto de los 5 temas de salud con más promesas. 


\section{DERECHOEIECTORAL}

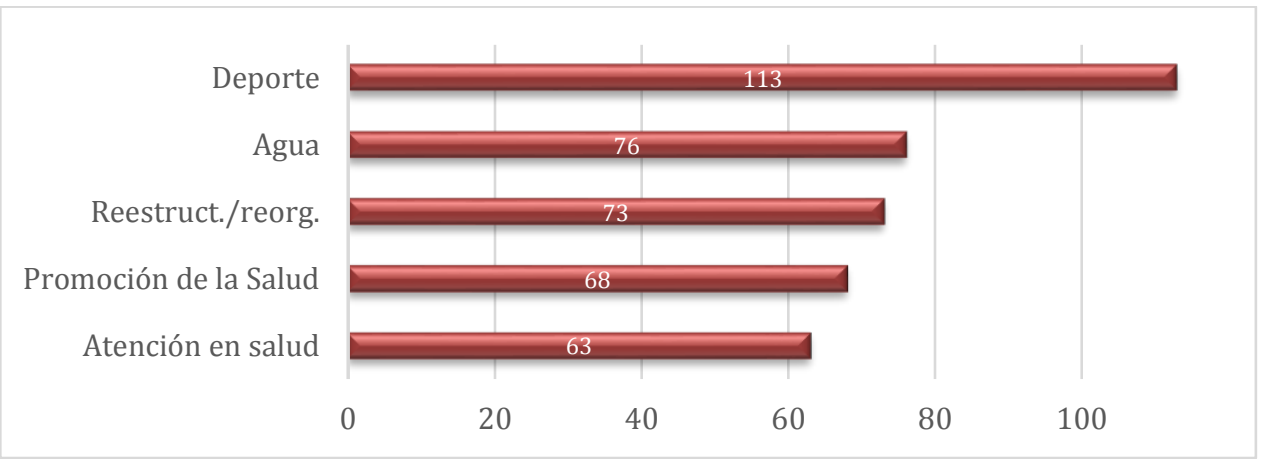

Figura 2. Promesas de políticas de mayor relevancia en el campo de la salud, elecciones presidenciales 2018, Costa Rica. Elaboración basada en los programas de gobierno.

Por otra parte, los énfasis temáticos de los partidos políticos difieren. Un análisis de los 5 partidos con mayor frecuencia de propuestas de política pública -que, dicho sea de paso, anuncian el $68 \%$ de todas las promesas en salud- arroja las siguientes áreas temáticas como prioritarias (solamente se presentan las 3 primeras):

- PAC: promoción de la salud (18), deporte (13), atención en salud (12), salud sexual reproductiva (12).

- PUSC: reestructuración/reorganización de los servicios de salud (34), finanzas de la CCSS (22), deporte (19).

- ML: deporte (31), atención en salud (16), formación continua (15).

- PLN: violencia intradomiciliaria (16), listas de espera (14), formación continua en salud (10).

- FA: gestión de residuos y reciclaje (22), agua (20), deporte (17).

Reagrupando los temas en categorías de primer orden, se pueden constatar los siguientes grandes componentes en materia de propuestas:

- Gestión en salud y prestación de los servicios de salud, con aspectos como la rectoría de salud, planificación, organización de los servicios, reestructuración de los servicios, mejora en la calidad de las prestaciones de los servicios, sistema de salud y recursos humanos sanitarios.

- Políticas de promoción de la salud, prevención de la salud, y la educación para la salud. 


\section{DERECHO EIECTORAL}

- Vialidad, lo que incluye políticas para aminorar los accidentes de tránsito y mejorar la transitabilidad por las ciudades.

- Políticas sobre sexualidad, esto incorpora a la salud sexual y a los derechos sexuales y/o reproductivos.

El núcleo de políticas agrupadas en "Gestión en salud y prestación de los servicios de salud" es el dominante de las propuestas (subyace en el 69\% de los 12 ejes temáticos dominantes). Lo interesante es que internamente la mayoría de las políticas públicas ofertadas refieren a reestructuraciones de los servicios de salud (73) y a mejoras propiamente en la atención de las personas aseguradas (63).

Aislando las propuestas sobre atención en salud (recuperación) y de los esquemas de organización de los servicios, hay otros 11 núcleos temáticos prioritarios en cuanto a promesas de políticas públicas, en las que sobresalen las de promoción/prevención/educación de la salud (146 políticas), deporte (113), vialidad (84) y agua (76). En la figura 3 se puede apreciar proporcionalmente el contenido de temas en propuestas de política.

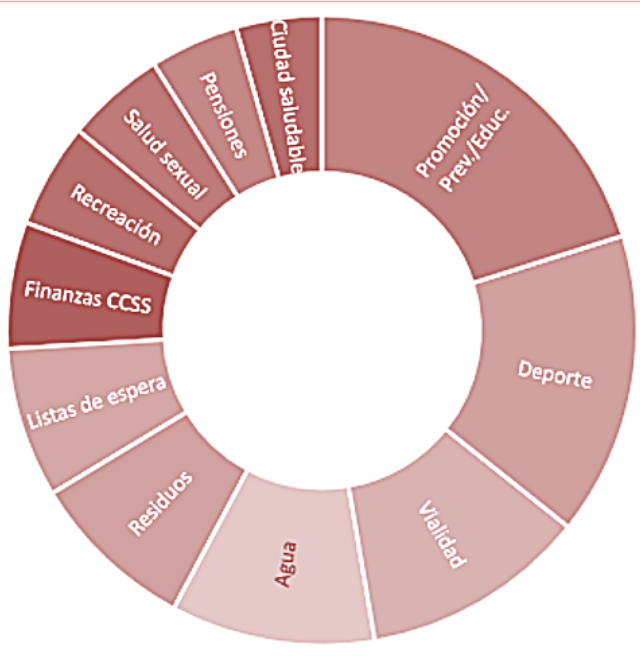

Figura 3. Agenda de política pública en salud de las principales promesas presentadas en la campaña electoral 2017-2018, a partir de núcleos temáticos, Costa Rica. Elaboración basada en los programas de gobierno.

Notas: Se excluyen los temas con menos de 30 políticas propuestas, y los propios del eje de "atención en salud y reorganización de los servicios". 


\section{DERECHO ELECTORAL}

No deja de ser relevante el hecho de que exista una cantidad importante de propuestas de políticas respecto del "recurso hídrico", las "listas de espera" en los servicios de salud, y la "vialidad".

Sobre los accidentes de tránsito, según datos del Consejo de Seguridad Vial, entre el año 2013 y el año 2016 la cantidad de muertes en sitio por accidentes de tránsito tuvo un crecimiento de $67,7 \%$, incluso la tasa de mortalidad en sitio por cada 100 mil habitantes fue de 6,32 en el 2013 y llega a 9,92 en el 2016 (Cosevi, 2016).

En cuanto a las listas de espera, la OCDE (2017) es del criterio de que, si bien la "cobertura universal de salud" es un logro, los pacientes esperan durante años por la atención definida. Indica, además, que los tiempos de espera para las cirugías electivas exceden el año. Por su parte, la Caja Costarricense de Seguro Social (CCSS, 2015) indicó que el tiempo de espera promedio para una cirugía general fue de 452 días; aproximadamente un $31 \%$ de los pacientes ha tenido una espera de 540 días. Las principales especialidades afectadas con estos tiempos de espera son los reemplazos de articulaciones, la extirpación de venas varicosas y las reparaciones de hernias inguinales. Los hospitales especializados son los más afectados con esta situación.

Resalta también la incorporación de políticas que son clasificables dentro del concepto de ciudades saludables, en tanto favorecen e impulsan la salud de la población. En la medida en que se relacionen con este concepto, se atiende a lo que señala el Consenso de Shanghái, en cuanto al compromiso de los Gobiernos locales que incluye el cumplimiento de los Objetivos del Desarrollo Sostenible, y el desarrollo de 5 principios de gobernanza, a saber: integrar la salud como consideración fundamental en todas las políticas; abordar todos los determinantes de la salud; promover una participación firme de la comunidad; reorientar los servicios sanitarios y sociales hacia la equidad; evaluar y vigilar el bienestar, la carga de morbilidad y los determinantes de la salud" (OMS, 2016).

Es destacable que las políticas públicas ofertadas en materia de promoción de la salud, prevención y educación para la salud sean numerosas. Es materia de otro análisis abordar qué promueven tales políticas, sus propósitos y el nivel de profundidad en las intervenciones que plantean.

Un análisis segmentado permite identificar los énfasis de propuestas elaborados por cada partido político. Por ejemplo, en materia de pensiones 


\section{DERECHO EIECTORAL}

el PUSC realiza 11 propuestas, mientras tanto hay 4 partidos sin propuesta alguna, a pesar de que en el período 2014-2017 fue un tema país el financiamiento y sostenibilidad del régimen de pensiones de la CCSS. En políticas de salud mental, el PLN realiza 8 propuestas, pero hay 11 partidos políticos sin una sola propuesta.

Si se toma en cuenta un problema público concreto como el de las "listas de espera", un tema propositivo como el de "educación/prevención/educación para la salud" y el de "finanzas de la CCSS", es posible palpar las diferencias de las propuestas de los partidos políticos (véase figura 4).

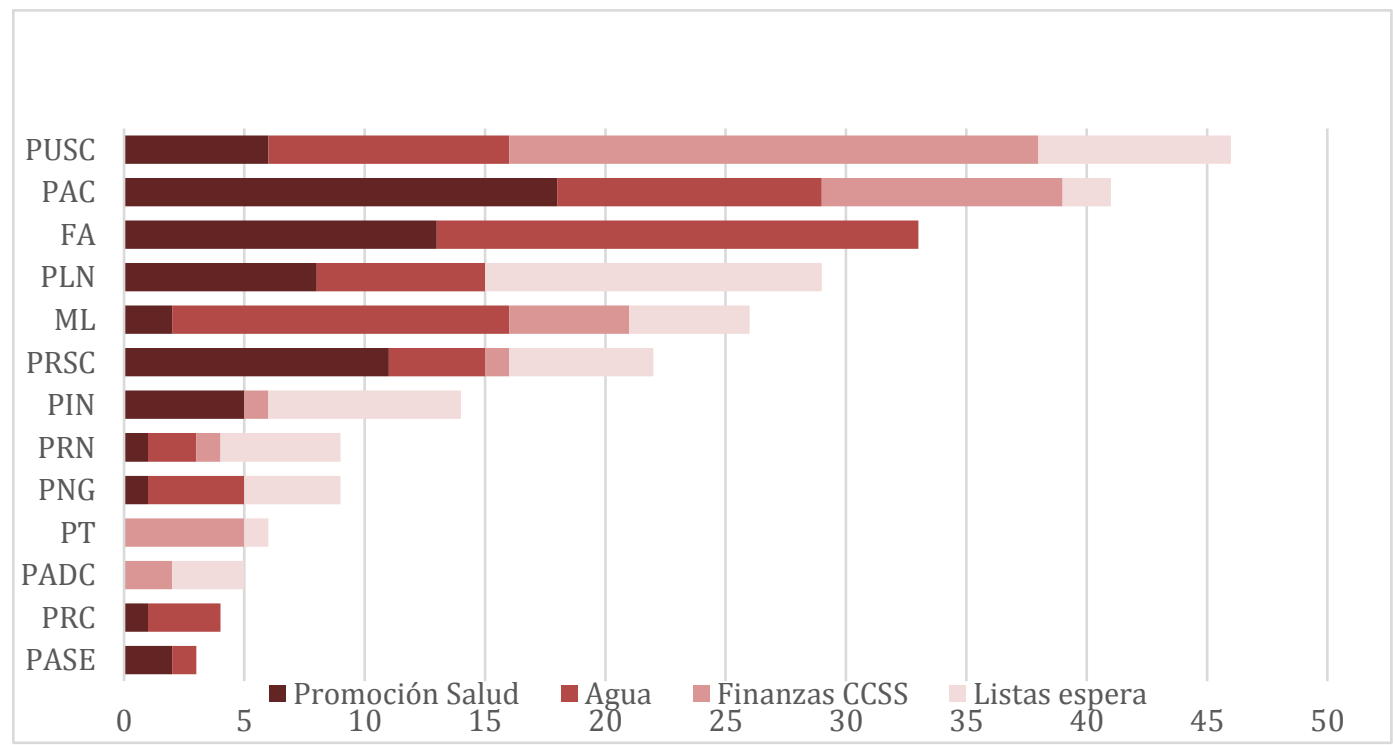

Figura 4. Comparación de ofertas de políticas sobre promoción de la salud, agua, finanzas de la CCSS, y listas de espera; elecciones presidenciales 2018, según partido político, Costa Rica. Elaboración basada en los programas de gobierno.

\subsection{LA PERSPECTIVA DE SALUd DE LOS PARTIDOS}

De los 13 partidos políticos solamente 3 (FA, PAC y PUSC) tienen una concepción de la salud que trasciende lo biológico y se inscribe en el marco de la producción social de la salud, esto es, de las condiciones sociales, políticas y económicas que, al incidir en una calidad de vida, afecta la salud de las personas. Posterior a la revisión de cada programa de gobierno se detecta: 


\section{DERECHO EIECTORAL}

a. El FA manifiesta situarse más allá de un concepto biológico de la salud (p. 83), abogando por una mejora en las condiciones de vida como parte del concepto de salud (p. 84); incluso, dice asentarse en el concepto de sistema de producción social de la salud (p. 84).

b. El PAC destaca el criterio de la "Promoción de la Salud como Derecho Humano, la prevención de enfermedades y el saneamiento básico local" (p. 146). En materia de los determinantes sociales de la salud establece la meta de elaborar un "Análisis de Situación de Salud Nacional" que se convierta en un "insumo para la evaluación y planificación de la producción social de la salud y los factores protectores, tomando en cuenta el agua potable, seguridad vial, educación en salud, nutrición, etc." (p. 147). En su oferta programática destaca cambios en el Ministerio de Salud, y de reforzamiento sobre la calidad de atención en los servicios de salud.

c. EI PUSC indica que "la política de salud es interdependiente con otras políticas sociales, razón por la cual implica un intenso trabajo intersectorial (...)" (p. 58); no obstante, el programa hace énfasis en la prestación de los servicios de salud. La violencia intrafamiliar, la atención del adulto mayor, las finanzas de la CCSS, y la importancia de la recreación como derecho humano son temas resaltados en la exposición programática.

Ahora bien, no siempre lo discursivo empata con el espíritu de lo propuesto, por ejemplo, en el caso del PUSC, las promesas tienen un énfasis sobre los servicios de salud de atención en un marco de intervención biológico, pese a su discurso oficial previamente mencionado.

Otros partidos políticos en sus programas de gobierno resaltan algunos temas o situaciones problemáticas en el ámbito de la salud que merecen intervención pública.

a. El PIN enfatiza sobre la protección y el fortalecimiento de la CCSS (p. 8). Dentro de los 7 temas que califica de urgentes en el país menciona dos que son propios de la salud: las pensiones y las listas de espera (p. 11).

b. El PRN aboga por lo que denomina como una "política preventiva y curativa universal"; resalta que "los enfoques puramente 'biologistas' de la salud deben sustituirse por enfoques integrales, en los que se 


\section{DERECHO ELECTORAL}

vincule con asertividad lo puramente físico con lo emocional" (p. 26), mas no se profundiza en políticas al respecto.

c. EI PASE desarrolla su programa de gobierno basado en 6 áreas de trabajo, donde una de ellas es la de "Atención y Promoción de la salud", pero la mayoría de tales propuestas se concentran en "estímulo al deporte".

d. EI PADC hace énfasis en el deterioro de los servicios de salud, indicando que se genera "por las restricciones presupuestarias que afectan la cantidad de personal, equipo e infraestructura, como por la inercia institucional que ha impedido una autoevaluación rigurosa (...)" (p. 48).

e. EI PNG puntualiza que "la situación que afronta el país en materia de salud pública es crítica" (p. 25); no obstante, el enfoque lo reduce al tema de listas de espera en los servicios de salud.

f. El PRS hace un llamado "a consolidar un pacto social en materia de salud y seguridad social" (p. 37); además, propone avanzar hacia un "modelo de Medicina Mixta Moderna sin fronteras"; y en la creación de un sistema nacional de salud, con un Ministerio de Salud que sí ejerza la rectoría que le compete.

g. El PML aboga por que cada ciudadano tenga la libertad de "elegir la atención médica y hospitalaria de su preferencia" (p. 74), y propone declarar como emergencia nacional "la crisis actual de las listas de espera, para poder realizar así los respectivos contratos de manera expedita" (p. 182).

h. EI PLN plantea varias políticas respecto de la violencia contra la mujer y la violencia infantil; sobre esta última se afirma que "es ya una epidemia en Costa Rica y requiere un abordaje integral, interinstitucional y bajo un enfoque de salud pública" (p. 47).

\section{Conclusiones}

En la oferta programática del proceso electoral presidencial 2018, en Costa Rica, es posible detectar más de mil promesas propias del ámbito de la salud (1052), donde el PAC y el PUSC presentaron una mayor cantidad (161 cada uno). 


\section{DERECHO ELECTORAL}

Algunas propuestas manifiestan trascender un concepto biologista de la salud en aras de un sistema de producción social de la salud, aunque parece más discursivo que real, ya que en la mayoría aparecen las promesas inconexas de una transversalidad o de un vínculo, por ejemplo, entre la salud y lo socioeconómico.

Se identificaron 75 temas propios de la salud, los cuales incluyen un amplio abanico de políticas, por ejemplo, derecho a la salud, atención al adulto mayor, adicciones, entre otros.

Los 5 temas con mayor número de propuestas son:

a. Políticas públicas sobre deporte.

b. Políticas respecto del recurso hídrico.

c. Políticas vinculadas con la reestructuración y reorganización de servicios, programas e instituciones del campo de la salud.

d. Políticas en promoción de la salud.

Si los temas se agrupan por afinidad, destacan las siguientes grandes categorías:

a. Propuestas en torno a la gestión en salud y la prestación de los servicios de salud.

b. Políticas de promoción de la salud, prevención y educación para la salud.

Hay diferencias conforme los partidos políticos, por ejemplo, el PAC enfatiza en promoción de la salud; el PUSC en la reestructuración/reorganización de los servicios de salud; el ML en deporte; el PLN en la violencia intradomiciliaria y el FA en gestión de residuos y reciclaje.

Según los resultados del análisis mostrado en este escrito, así como de los propiamente electorales de la segunda ronda, podría inferirse que le corresponde al PAC durante la gestión de gobierno (2018-2021) abordar problemas públicos relacionados con la promoción de la salud, el deporte, la atención en salud y la salud sexual y reproductiva.

En ese sentido, resulta imperioso analizar cómo se concretan esas promesas políticas realizadas en el ámbito de la salud, de manera que corresponda con la confianza que el electorado mostró en las urnas para llevar adelante las propuestas de gobierno. 


\section{DERECHO ELECTORAL}

La campaña del TSE, "Un voto informado", es una estrategia valiosa para incentivar al electorado para el reconocimiento y estudio de la perspectiva de salud de cada partido político, entre otras áreas. Es imprescindible el diseño de alguna política para el seguimiento y diseminación de los cumplimientos, ya que es parte de una ciudadanía activa.

En otras investigaciones se puede profundizar en:

a. El enfoque sobre temas en particular, por ejemplo, en listas de espera o en reorganización de los servicios de salud; esto es, si existen diferencias de fondo respecto del alcance y la forma efectiva de solucionarlos o abordarlos.

b. La hechura de las metas que tienen las políticas propuestas, ya que en la presentación de los programas de gobierno se carece de una estructura obligatoria del tipo problema-propuesta-meta.

c. La materialización de las promesas del partido político ganador en las elecciones 2018 en el Plan Nacional de Desarrollo.

d. Si como ente rector de la salud, el Ministerio de Salud debe dar algún seguimiento y apoyo al cumplimiento de las promesas de salud esbozadas por el partido político ganador.

\section{REFERENCIAS BIBLIOGRÁFICAS}

Corte Interamericana de Derechos Humanos. (2017). Opinión consultiva OC-24/17, 24/11/2017, solicitada por la República de Costa Rica, identidad de género, e igualdad y no discriminación a parejas del mismo sexo. Documento. San José, C.R.: CIDH.

Costa Rica. Consejo de Seguridad Vial (2016). Observatorio de seguridad vial, estadísticas. Recuperado de https://www.csv.go.cr/estadisticas

Costa Rica. Caja Costarricense de Seguro Social (2015). Evaluación 2014. Prestación de Servicios de Salud. Áreas de Salud, Hospitales y Centros Especializados. Recuperado de http://www.binasss.sa.cr/serviciosdesalud/2014.pdf

Costa Rica. Tribunal Supremo de Elecciones (2018). Resultados definitivos 4 de febrero de 2018.2 Recuperado de http://resultados2018.tse.go.cr/resultadosdefinitivos/\#/presidenciales

Díaz, J. A. (2018). El gobierno del bicentenario en Costa Rica. Foreign Affairs Latinoamérica, Vol. 18 (4), 37-44. Recuperado de www.fal.itam.mx 


\section{DERECHO EIECTORAL}

Estado de la Nación (2017). Informe Estado de La Nación 2017. Recuperado de https://www.estadonacion.or.cr/2017

La Nación. Ministro de Deportes anuncia teletón para financiar atletas. Crónica. San José, C.R. 14 de julio, 2011. Recuperado de https://www.nacion.com/purodeporte/otros-deportes/ministro-de-deportes-anuncia-teleton-parafinanciar-atletas

La Revista. (2017). Un voto informado es un voto inteligente iSea responsable con Costa Rica! Recuperado de https://www.larevista.cr/voto-informado-votointeligente/

Organización para la Cooperación y el Desarrollo Económicos (2017). Assessment and recommendations. En OECD Reviews of Health Systems: Costa Rica, OECD Publishing, París. Recuperado de https://www.ministeriodesalud.go.cr/

Organización Mundial de la Salud (2016). Consenso de Shanghái sobre Ciudades Saludables. Recuperado de http://www.who.int/healthpromotion/conferences/9gchp/mayors-consensusES.pdf

Partido Accesibilidad sin Exclusión (Costa Rica) (2017). Plan de Gobierno 2018-2022. Hacia una nueva Constitución Política. San José. C.R.: Accesibilidad sin Exclusión.

Partido Acción Ciudadana (Costa Rica) (2017). Carlos 2018. Creer y crear la Costa Rica del siglo XXI: compromiso país. San José. C.R.: Acción Ciudadana.

Partido Alianza Demócrata Cristiana (Costa Rica) (2017). Plan de Gobierno 2018-2022. Hagamos de Costa Rica un país de oportunidades. San José. C.R.: Alianza Democrática.

Partido Frente Amplio (Costa Rica) (2017). Plan de Gobierno 2018-2022. Un país equilibrado para que nadie se quede atrás. San José. C.R.: Frente Amplio.

Partido Integración Nacional (Costa Rica) (2017). Costa Rica el país azul. Perspectivas para la reconstrucción nacional. Administración Juan Diego Castro Fernández (2018-2022). San José. C.R.: Integración Nacional.

Partido Liberación Nacional (Costa Rica) (2017). El momento es ahora. Álvarez Desanti. San José. C.R.: Liberación Nacional.

Partido Movimiento Libertario (Costa Rica) (2017). Gobernar por Costa Rica: programa de gobierno. San José. C.R.: Movimiento Libertario. 


\section{DERECHO EIECTORAL}

Partido Nueva Generación (Costa Rica) (2017). Sergio 2018. Propuesta de Gobierno 2018-2022. San José. C.R.: Nueva Generación.

Partido Renovación Costarricense (Costa Rica) (2017). Un país comprometido con el bienestar de las familias. Propuesta política 2018-2022. San José. C.R.: Renovación Costarricense.

Partido Republicano Social Cristiano (Costa Rica) (2017). Programa de Gobierno. Humanismo, Libertad y Justicia. Versión resumida 2018-2022. San José. C.R.: Republicano Social Cristiano.

Partido Restauración Nacional (Costa Rica) (2017). Costa Rica iHagámoslo juntos! Plan de Gobierno 2018-2022. San José. C.R.: Restauración Nacional.

Partido De Los Trabajadores (Costa Rica) (2017). Programa socialista para Un Gobierno de los Trabajadores. 2018-2022. San José. C.R.: De los Trabajadores.

Partido Unidad Social Cristiana (Costa Rica) (2017). Aquí sí hay algo por qué votar. Plan de Gobierno. Vote por algo. Piza Presidente. San José. C.R.: Unidad Social Cristiana.

Rosales, R. (2018). En: Adenauer, K. Fundación. Nuevas campañas electorales en América Latina. Costa Rica: volatilidad, fragmentación, shock religioso y decisiones de último minuto. 55-67. Recuperado de: https://dialogopolitico.org/wp-content/uploads/2019/01/Campa\%C3\% B1as-electorales-en-Am\%C3\%A9rica-Latina_WEB.pdf

Semanario Universidad. (2018). Entrevista. Luis Antonio Sobrado, presidente del TSE "Es importante de ir a votar libres de presiones religiosas". Recuperado de https://semanariouniversidad.com/pais/importante-ir-votar-librespresiones-religiosas/ 